\title{
Performance Analysis of Diffusion-Based Molecular Communications With Memory
}

\author{
Sebastià Galmés and Baris Atakan
}

\begin{abstract}
In this paper, the comprehensive delay and performance analyses of the $M$-ary molecular communications with memory are presented. By taking into account any level of channel memory, the type-based and concentration-based modulation schemes are introduced and analyzed. In the typebased modulation, information symbols are encoded through different molecule types. In the concentration-based modulation, various concentration levels of one molecule type are used to encode information symbols. For both modulation schemes, the delay distributions of the molecular symbols are derived, and then, the symbol error probabilities are developed. The given distributions and the error probability expressions are validated through extensive simulation experiments. After showing that the derived expressions are valid, the performance of the modulation schemes is evaluated. The performance evaluations reveal that by properly selecting the parameters such as slot time and number of emitted molecules, the performance can be improved in both type and concentration-based molecular communication as the channel memory is increased. Furthermore, it is shown that the type-based molecular communication outperforms the concentration-based molecular communication.
\end{abstract}

Index Terms-Diffusion-based molecular communication, delay distribution, symbol error probability, counting process.

\section{INTRODUCTION}

$\mathbf{R}$ APID development in nano and biotechnology allows the fabrication of NanoMachines (NMs). Because of their limited capabilities, they can only accomplish a simple specific task in a scale ranging from 1 to 100 nanometers. However, the communication of NMs in a nanonetwork can extend their capabilities to realize many sophisticated applications. Such nanonetworks can also increase the number of design possibilities with highly sophisticated behaviors. Molecular Communication (MC) in which messenger molecules are used to share information is a promising alternative for the interconnection of NMs in nanonetworks [1]-[7].

In this paper, we consider two basic MC schemes:

- M-Level Molecular Shift Keying (M-MOSK): M different information symbols are carried by $\mathrm{M}$ different

Manuscript received July 14, 2015; revised November 26, 2015, March 19, 2016, and July 5, 2016; accepted July 11, 2016. Date of publication July 20, 2016; date of current version September 14, 2016. This work was supported by the Turkish Scientific and Technological Research Council (TUBITAK) under Grant 115E362. The associate editor coordinating the review of this paper and approving it for publication was M. Pierobon.

S. Galmés is with the Department of Mathematics and Computer Science, University of Balearic Islands, Palma 07122, Spain (e-mail: sebastia.galmes@uib.es).

B. Atakan is with the Department of Electrical and Electronics Engineering, İzmir Institute of Technology, İzmir 35430, Turkey (e-mail: barisatakan@iyte.edu.tr).

Color versions of one or more of the figures in this paper are available online at http://ieeexplore.ieee.org.

Digital Object Identifier 10.1109/TCOMM.2016.2593469 types of molecules emitted by the transmitter nanomachine (TN). The detection of symbols is assumed whenever the number of molecules that hit the receiver nanomachine (RN) during a slot time is above a predefined threshold.

- M-Level Concentration Shift Keying (M-CSK): A single type of molecule is used, and there are $\mathrm{M}$ different emission levels to generate $\mathrm{M}$ different symbols. Correct detection of a symbol occurs whenever the number of molecules hitting the $\mathrm{RN}$ is between the predefined thresholds associated to that symbol.

A bunch of related works can be introduced as follows. In [8] and [9], two different modulation schemes called concentration shift keying and molecule shift keying are introduced. The symbol error probabilities of these schemes are derived in order to quantify their performances. In these works, one-slot memory is considered, which means that each transmission is assumed to be affected by only previous transmission. Therefore, the given analysis cannot quantify the performance of MC with arbitrary level of memory. In [10], the fourth-order channel memory is investigated and the corresponding bit error probabilities are derived. Since the level of the channel memory is set to four, the given analysis is not extensible to arbitrary level of memory case. Furthermore, the delay distribution of the symbols is not derived in this reference. In [11] and [12], an elegant physicchemical model is introduced to characterize the combined effects of transmitter, transmission medium and receiver as a transfer function between input and output. It is assumed that consecutive symbols are well separated in time so that inter-symbol interference (ISI) can be neglected. Therefore, the effects of ISI through arbitrary level of memory cannot be discussed in this work. In [13] and [14], a Poisson model is used for the flow of molecules exiting the storage and diffusing in the environment. In order to take into account the overall effect of previous time slots, a compound Poisson process is derived as a weighted sum of the Poisson processes corresponding to previous slots. While the symbol error probability is derived for an arbitrary level of memory, the probability distributions of symbols are not investigated in this work. In general, our contribution over the existing works can be briefed as follows:

- We derive the probability distributions of the symbol delay and then, we derive the symbol error probability for the M-MOSK and M-CSK schemes.

- We consider the general multi-level signaling case for the M-MOSK and M-CSK schemes. 
- We determine the memory effects for both modulation schemes in an exact way, and provide an algorithm to speed up the computation of these effects.

The remainder of the paper is organized as follows. The type-based molecular scheme is first considered in Section II. In Section II-A, an efficient algorithm is introduced to speed up the computations of the derived probability distribution. In Section III, the concentration-based molecular scheme is investigated. Then, the derived probability distributions and the symbol error probabilities are validated through extensive simulation experiments in Section IV. Finally, the performance evaluations are introduced in Section VI and the concluding remarks are discussed in Section VII.

\section{M-Level Molecular Shift Keying (M-MOSK) WITH $m$-SLOT MEMORY}

We consider an $M$-ary molecular communication (MC) scheme in which each symbol $S_{a}, a \in\{1, \ldots, M\}$ is transmitted by emitting $N_{a}$ molecules of type $\psi_{a}$. This emission is performed by the TN at the beginning of a time slot. The symbol is correctly received if at least $\sigma_{a}$ molecules of type $\psi_{a}$ reach the RN within a slot duration. Otherwise, the symbol is undetected. Each type of molecule has its own diffusion coefficient and emission level parameters $\left(N_{a}, \sigma_{a}\right)$.

Let us first define the counting process $c_{a}\left(N_{a}, t\right)$, which stands for the number of molecules of type $\psi_{a}$ that have hit the RN until time $t$, given that $N_{a}$ molecules were emitted at the beginning of slot $n(t=0)$. The probability mass function (PMF) associated with this process, $\varphi_{a}\left(N_{a}, l, t\right)$, is the probability that exactly $l$ molecules have reached the $\mathrm{RN}$ at time $t$, given that a set of $N_{a}$ molecules were emitted at the beginning of slot $n$. In fact, it is a binomial distribution given as

$$
\begin{aligned}
\varphi_{a}\left(N_{a}, l, t\right) & =\operatorname{Pr}\left(c_{a}\left(N_{a}, t\right)=l\right) \\
& =\left(\begin{array}{c}
N_{a} \\
l
\end{array}\right) F_{\psi_{a}}^{l}(t)\left[1-F_{\psi_{a}}(t)\right]^{N_{a}-l},
\end{aligned}
$$

where $l \in\left[0, N_{a}\right], t \geq 0$ and $F_{\psi_{a}}(t)$ is the cumulative distribution function of the delay experienced by any single molecule of type $\psi_{a}$ to reach the RN. It is given as [15]

$$
F_{\psi_{a}}(t)=\operatorname{erfc}\left(\frac{d}{2 \sqrt{D_{\psi_{a}} t}}\right), \quad t>0,
$$

where $D_{\psi_{a}}$ is the diffusion coefficient of molecule type $\psi_{a}$ and $d$ is the distance between the TN and RN.

Let us also consider a modified counting process, $c_{a, i, h}\left(N_{a}, t\right)$ to register the molecules corresponding to symbol $S_{a}$ that are emitted at slot $n-i$ and hit the RN during a time interval $t$ starting at the beginning of slot $\mathrm{n}$, given that the emission level is $N_{a}$ and a number of $h\left(0 \leq h \leq N_{a}\right)$ molecules have already reached the RN during slot $n-i$. Then, the PMF associated with $c_{a, i, h}\left(N_{a}, t\right)$ can be formulated as the probability that $h+j$ molecules have hit the $\mathrm{RN}$ at time $t+\tau$, given that $h$ molecules have already reached the RN during previous slot duration $i \tau$. This PMF function is denoted by $\varphi_{a, i, h}\left(N_{a}, j, t\right)$ and derived in (3), as shown at the bottom of this page. We can now un-condition this probability with respect to $h$ in order to characterize the counting process $c_{a, i}\left(N_{a}, t\right)$, which stands for the amount of molecules of type $\psi_{a}$ from the previous slot that hit the $\mathrm{RN}$ in the interval of duration $t$ starting at the beginning of the current slot. The uncondioned PMF, i.e., $\varphi_{a, i}\left(N_{a}, j, t\right)$, is given in (4), as shown at the bottom of this page. The delay experienced by a symbol transmitted in a certain slot depends on the sequence of symbols transmitted in the $m$ previous slots. In order to develop a general formulation, we can introduce a new counting process associated with every previous slot, called, $e_{a, i}\left(N_{a}, t\right), i=1, \ldots, m$, to denote the amount of molecules of type $\psi_{a}$ transferred from previous slot $n-i$ to the current slot (see Figure 1). This counting process can be characterized as follows

$$
e_{a, i}\left(N_{a}, t\right)= \begin{cases}c_{a, i}\left(N_{a}, t\right), & s(n-i)=S_{a} \\ 0, & s(n-i)=S_{b}, \quad b \neq a,\end{cases}
$$

$$
\begin{aligned}
\varphi_{a, i, h}\left(N_{a}, j, t\right)= & \operatorname{Pr}\left(c_{a, i, h}\left(N_{a}, t\right)=j\right)=\operatorname{Pr}\left(c_{a}\left(N_{a}, t+i \tau\right)=h+j \mid c_{a}\left(N_{a}, i \tau\right)=h\right) \\
= & \frac{\operatorname{Pr}\left(c_{a}\left(N_{a}, t+i \tau\right)=h+j, c_{a}\left(N_{a}, i \tau\right)=h\right)}{\operatorname{Pr}\left(c_{a}\left(N_{a}, i \tau\right)=h\right)} \\
= & \frac{\left(\begin{array}{c}
N_{a} \\
h
\end{array}\right)\left(\begin{array}{c}
N_{a}-h \\
j
\end{array}\right) F_{\psi_{a}}^{h}(\tau)\left[F_{\psi_{a}}(t+i \tau)-F_{\psi_{a}}(i \tau)\right]^{j}\left[1-F_{\psi_{a}}(t+i \tau)\right]^{N_{a}-(h+j)}}{\left(\begin{array}{c}
N_{a} \\
h
\end{array}\right) F_{\psi_{a}}^{h}(i \tau)\left[1-F_{\psi_{a}}(i \tau)\right]^{N_{a}-h}} \\
= & \left(\begin{array}{c}
N a-h \\
j
\end{array}\right) \frac{\left[F_{\psi_{a}}(t+i \tau)-F_{\psi_{a}}(i \tau)\right]^{j}\left[1-F_{\psi_{a}}(t+i \tau)\right]^{N_{a}-(h+j)}}{\left[1-F_{\psi_{a}}(i \tau)\right]^{N_{a}-h}}, \\
\varphi_{a, i}\left(N_{a}, j, t\right)= & \operatorname{Pr}\left(c_{a, i}\left(N_{a}, t\right)=j\right)=\sum_{h=0}^{N_{a}-j} \operatorname{Pr}\left(c_{a, i, h}\left(N_{a}, t\right)=j\right) \operatorname{Pr}\left(c_{a}\left(N_{a}, i \tau\right)=h\right) \\
= & \sum_{h=0}^{N_{a}-j}\left(\begin{array}{c}
N_{a} \\
h
\end{array}\right)\left(\begin{array}{c}
N_{a}-h \\
j
\end{array}\right) F_{\psi_{a}}^{h}(i \tau)\left[F_{\psi_{a}}(t+i \tau)-F_{\psi_{a}}(i \tau)\right]^{j}\left[1-F_{\psi_{a}}(t+i \tau)\right]^{N_{a}-(h+j)} \\
= & {\left[F_{\psi_{a}}(t+i \tau)-F_{\psi_{a}}(i \tau)\right]^{j} \sum_{h=0}^{N_{a}-j}\left(\begin{array}{c}
N_{a} \\
h
\end{array}\right)\left(\begin{array}{c}
N_{a}-h \\
j
\end{array}\right) F_{\psi_{a}}^{h}(i \tau)\left[1-F_{\psi_{a}}(t+i \tau)\right]^{N_{a}-(h+j)}, }
\end{aligned}
$$




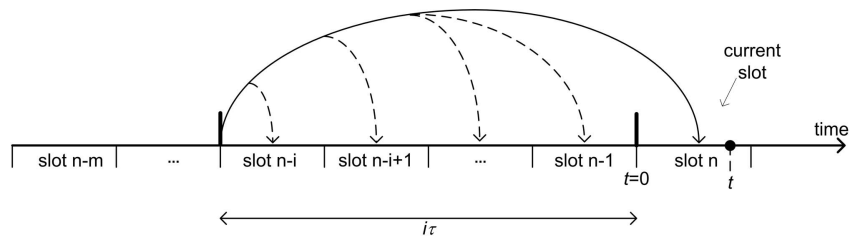

Fig. 1. Influence of slot $n-i$ on slot $n$ in the $m$-memory case.

where $s(n-i)$ denotes the symbol transmitted at slot $n-i$. The PMF of $e_{a, i}\left(N_{a}, t\right)$, i.e., $\tilde{\varphi}_{a, i}\left(N_{a}, j, t\right)$, is given by

$$
\begin{aligned}
\tilde{\varphi}_{a, i} & \left(N_{a}, j, t\right)=\operatorname{Pr}\left(e_{a, i}\left(N_{a}, t\right)=j\right) \\
= & {\left[\frac{1}{M} \operatorname{Pr}\left(c_{a, i}\left(N_{a}, t\right)=j\right)+\frac{M-1}{M} \delta(j)\right] } \\
= & {\left[\frac{1}{M} \varphi_{a, i}\left(N_{a}, j, t\right)+\frac{M-1}{M} \delta(j)\right], }
\end{aligned}
$$

where $j=0, \ldots, N_{a}$ and $\delta($.$) denotes the discrete delta$ function. The compound counting process, i.e., $c_{a, T, m}\left(N_{a}, t\right)$, resulting from $m$ previous slots and the current slot can now be formulated in the following way:

$$
c_{a, T, m}\left(N_{a}, t\right)=\left(\sum_{i=1}^{m} e_{a, i}\left(N_{a}, t\right)\right)+c_{a}\left(N_{a}, t\right)
$$

Since the individual counting processes contained in (7) are mutually independent (because they correspond to different time slots), again the convolution with respect to $k$ is suited to describe the PMF of $c_{a, T, m}\left(N_{a}, t\right)$ :

$$
\begin{aligned}
\varphi_{a, T, m}\left(N_{a}, k, t\right)=\varphi_{a}\left(N_{a}, k, t\right) & \\
& *\left[\tilde{\varphi}_{a, 1}\left(N_{a}, k, t\right) * \ldots * \tilde{\varphi}_{a, m}\left(N_{a}, k, t\right)\right]
\end{aligned}
$$

where $k=0, \ldots,(m+1) N_{a}$. Then, the distribution function of the delay experienced by symbol $S_{a}$ can be formulated as

$$
\begin{aligned}
F_{a}(t) & =\operatorname{Pr}\left(\gamma_{a} \leq t\right)=\operatorname{Pr}\left(c_{a, T, m}\left(N_{a}, t\right) \geq \sigma_{a}\right) \\
& =\sum_{k=\sigma_{a}}^{(m+1) N_{a}} \varphi_{a, T, m}\left(N_{a}, k, t\right)
\end{aligned}
$$

By taking the derivative with respect to time, the probability density function $f_{a}(t)$ can be obtained. By using $F_{a}(t)$ in (9), the probability that the symbol $S_{a}$ is delivered to the receiver within the current slot can be given as $F_{a}(\tau)$. However, no other symbol should be delivered within the same slot to ensure that the RN just receives symbol $S_{a}$. Therefore, it should be also checked whether or not other symbols are delivered within the same slot. To this end, let us focus on symbol $S_{b}$ with $b \neq a$. We know that $S_{b}$ is not transmitted in the current slot. However, it is likely that $S_{b}$ has been transmitted during the past $m$ slots. Thus, possible arrivals of molecules $\psi_{b}$ to the RN within the current slot need to be investigated. Similar to $e_{a, i}\left(N_{a}, t\right)$ in (5), the counting process $e_{b, i}\left(N_{b}, t\right)$ characterizes the amount of molecules of type $\psi_{b}$ transferred from previous slot $n-i$. It can be defined as follows:

$$
e_{b, i}\left(N_{b}, t\right)= \begin{cases}c_{b, i}\left(N_{b}, t\right), & s(n-i)=S_{b} \\ 0, & s(n-i)=S_{a}, \quad a \neq b,\end{cases}
$$

where $c_{b, i}\left(N_{b}, t\right)$ is the extension of counting process $c_{a, i}\left(N_{a}, t\right)$ for symbol $S_{b}$. In order to obtain the PMF corresponding to $c_{b, i}\left(N_{b}, t\right)$, i.e., $\varphi_{b, i}\left(N_{b}, j, t\right)$, it is simply sufficient to substitute $N_{b}$ and $F_{\psi_{b}}($.$) instead of N_{a}$ and $F_{\psi_{a}}($.$) in (4). Similar to (6), the PMF of e_{b, i}\left(N_{a}, t\right)$, i.e., $\tilde{\varphi}_{b, i}\left(N_{b}, j, t\right)$, is

$$
\begin{aligned}
\tilde{\varphi}_{b, i} & \left(N_{b}, j, t\right)=\operatorname{Pr}\left(e_{b, i}\left(N_{b}, t\right)=j\right) \\
= & {\left[\frac{1}{M} \operatorname{Pr}\left(c_{b, i}\left(N_{b}, t\right)=j\right)+\frac{M-1}{M} \delta(j)\right] } \\
= & {\left[\frac{1}{M} \varphi_{b, i}\left(N_{b}, j, t\right)+\frac{M-1}{M} \delta(j)\right], }
\end{aligned}
$$

where $j=0, \ldots, N_{b}$. For symbol $S_{b}$, the compound counting process resulting from $m$ previous slots is

$$
\tilde{c}_{b, T, m}\left(N_{b}, t\right)=\sum_{i=1}^{m} e_{b, i}\left(N_{b}, t\right) .
$$

Similar to (8), the PMF of this compound process:

$$
\tilde{\varphi}_{b, T, m}\left(N_{b}, k, t\right)=\tilde{\varphi}_{b, 1}\left(N_{b}, k, t\right) * \ldots * \tilde{\varphi}_{b, m}\left(N_{b}, k, t\right) .
$$

Then, the distribution function of the delay experienced by symbol $S_{b}$ can be formulated in this way:

$$
\begin{aligned}
F_{b}(t) & =\operatorname{Pr}\left(\gamma_{b} \leq t\right)=\operatorname{Pr}\left(\tilde{c}_{b, T, m}\left(N_{b}, t\right) \geq \sigma_{b}\right) \\
& =\sum_{k=\sigma_{b}}^{m N_{b}} \tilde{\varphi}_{b, T, m}\left(N_{b}, k, t\right),
\end{aligned}
$$

where $\gamma_{b}$ is the delay experienced by symbol $S_{b}$. By using $F_{b}(t)$ in (14), the probability that symbol $S_{b}$ is delivered to the RN within the current slot can be introduced as $F_{b}(\tau)$. Now, we can write the probability that symbol $S_{a}$ is the only symbol which is successfully delivered to the $\mathrm{RN}$ as follows:

$$
P_{a}=F_{a}(\tau) \prod_{b \neq a}\left[1-F_{b}(\tau)\right] .
$$

The symbol error probability for symbol $S_{a}$ can be given as

$$
\operatorname{SEP}_{a}=1-P_{a} .
$$

By taking into account all symbol types, the average symbol error probability can be written as

$$
\mathrm{SEP}=\frac{1}{M} \sum_{a=1}^{M} \operatorname{SEP}_{a}
$$

\section{A. Efficient Algorithm for the Evaluation of Expression (4)}

A recursive formulation which speeds up the evaluation of expression (4) is developed in (18), as shown at the bottom of the next page. This derivation relies on a complementary result which is given in (19), as shown at the bottom of the next page. To implement the recursion given by (18), some particular results of $\varphi_{a, i}\left(n_{a}, j, t\right)$ are especially interesting, where $n_{a}$ is an auxiliary variable that denotes the current value of the emission level:

$$
\begin{aligned}
\varphi_{a, i}\left(n_{a}, 0, t\right) & =\left[1-\left(F_{\psi_{a}}(t+i \tau)-F_{\psi_{a}}(i \tau)\right)\right]^{n_{a}} \\
\varphi_{a, i}\left(n_{a}, n_{a}, t\right) & =\left[F_{\psi_{a}}(t+i \tau)-F_{\psi_{a}}(i \tau)\right]^{n_{a}}
\end{aligned}
$$




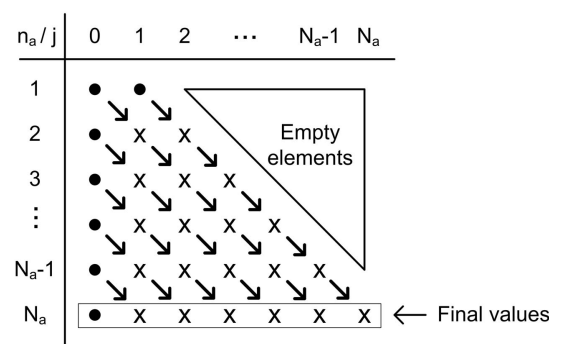

Fig. 2. The illustration of how the recursive formula in (18) is implemented.

The matrix given in Fig. 2 can be introduced which reflects all combinations of the two magnitudes and allows for a straightforward representation of the recursion process: In this matrix, the arrows show the evolution of the iteration process from the initial values represented as dots. Also, note that the upper triangle of the matrix is empty, because $j$ cannot be above $n_{a}\left(0 \leq j \leq n_{a}, n_{a}=1, \ldots, N_{a}\right)$.

The elements in the first column of the matrix in Fig. 2 can be easily obtained according to (20), whereas the element in position $(1,1)$ in the matrix can be derived from $(21)$ :

$$
\begin{aligned}
\varphi_{a, i}(1,1, t) & =F_{\psi_{a}}(t+i \tau)-F_{\psi_{a}}(i \tau) \\
& =1-\varphi_{a, i}(1,0, t)
\end{aligned}
$$

Thus, elements $(0,1)$ and $(1,1)$ in the matrix given in Fig. 2 are complementary with respect to 1 . It will be enough to use a single vector of dimension $N_{a}+1$ initialized with the dot elements in the matrix, and then apply the iteration process as many times as necessary until the last row in the matrix is obtained. This is repeated for every $i=1, \ldots, m$ with $t=\tau$, which means that only the values $F_{\psi_{a}}(\tau), \ldots, F_{\psi_{a}}((m+1) \tau)$ need to be evaluated during the setup process.

\section{M-Level Concentration ShIFt Keying OR (M-CSK) WITH $m$-SLOT MEMORY}

In this case, we consider an $\mathrm{M}$-ary molecular communication scheme in which each symbol $S_{a}$, with $a \in\{1, \ldots, M\}$, is transmitted by emitting $N_{a}$ molecules of type $\psi$ at the beginning of a time slot. The RN uses a set of thresholds $\left\{\sigma_{1}, \ldots, \sigma_{M}\right\}$ to detect the symbols, meaning that a symbol is correctly received if the amount of molecules that reach the RN after a time slot is between its corresponding thresholds.

As before, expression (7) describes the compound counting process, though slightly modified to reflect the fact that the transfer variable, now denoted as $e_{i}(t)$, is generally non-zero whatever symbol is transmitted in slot $n-i$ :

$$
c_{a, T, m}\left(N_{a}, t\right)=\left(\sum_{i=1}^{m} e_{i}(t)\right)+c_{a}(t)
$$

Specifically, the transfer variables can now be given by

$$
e_{i}(t)=\sum_{b=1}^{M} c_{b, i}\left(N_{b}, t\right) \times \delta\left(s(n-i), S_{b}\right) .
$$

Here, the Kronecker delta has been introduced in order to obtain 1 when $s(n-i)=S_{b}$ and 0 otherwise. Expression (24) shows that interference can now be produced by any previous symbol since the $\mathrm{TN}$ emits a single type of molecules, i.e., $\psi$. Moreover, $c_{b, i}\left(N_{b}, t\right)$ is characterized by expression (4), although now a single type of molecule is used.

$$
\begin{aligned}
\varphi_{b, i}\left(N_{b}, j, t\right)= & \operatorname{Pr}\left(c_{b, i}\left(N_{b}, t\right)=j\right) \\
= & {\left[F_{\psi}(t+i \tau)-F_{\psi}(i \tau)\right]^{j} } \\
& \times \sum_{h=0}^{N_{b}-j}\left(\begin{array}{c}
N_{b} \\
h
\end{array}\right)\left(\begin{array}{c}
N_{b}-h \\
j
\end{array}\right) F_{\psi}^{h}(i \tau) \\
& \times\left[1-F_{\psi}(t+i \tau)\right]^{N_{b}-(h+j)},
\end{aligned}
$$

where $0 \leq j \leq N_{b}, 1 \leq b \leq M, 1 \leq i \leq m$ and $t \geq 0$. Again, the algorithm proposed in subsection II-A can be used in order to speed up the computation of these probabilities.

$$
\begin{aligned}
& \varphi_{a, i}\left(N_{a}, j+1, t\right)=\operatorname{Pr}\left(c_{a, i}\left(N_{a}, t\right)=j+1\right) \\
& =\left[F_{\psi_{a}}(t+i \tau)-F_{\psi_{a}}(i \tau)\right]^{j+1} \sum_{h=0}^{N_{a}-(j+1)}\left(\begin{array}{c}
N_{a} \\
h
\end{array}\right)\left(\begin{array}{c}
N_{a}-h \\
j+1
\end{array}\right) \\
& \times F_{\psi_{a}}^{h}(i \tau)\left[1-F_{\psi_{a}}(t+i \tau)\right]^{N_{a}-(h+j+1)} \\
& =\left[F_{\psi_{a}}(t+i \tau)-F_{\psi_{a}}(i \tau)\right]\left[F_{\psi_{a}}(t+i \tau)-F_{\psi_{a}}(i \tau)\right]^{j} \\
& \times \sum_{h=0}^{\left(N_{a}-1\right)-j} \frac{N_{a}}{j+1}\left(\begin{array}{c}
N_{a}-1 \\
h
\end{array}\right)\left(\begin{array}{c}
N_{a}-1-h \\
j
\end{array}\right) F_{\psi_{a}}^{h}(i \tau)\left[1-F_{\psi_{a}}(t+i \tau)\right]^{N_{a}-1-(h+j)} \\
& =\left[F_{\psi_{a}}(t+i \tau)-F_{\psi_{a}}(i \tau)\right] \frac{N_{a}}{j+1} \varphi_{a, i}\left(N_{a}-1, j, t\right) \\
& \left(\begin{array}{c}
N_{a} \\
h
\end{array}\right)\left(\begin{array}{c}
N_{a}-h \\
j+1
\end{array}\right)=\left(\begin{array}{c}
N_{a} \\
h
\end{array}\right) \frac{\left(N_{a}-h\right) !}{(j+1) !\left(N_{a}-h-j-1\right) !}=\frac{1}{j+1}\left(\begin{array}{c}
N_{a} \\
h
\end{array}\right) \frac{\left(N_{a}-h\right) !}{j !\left(N_{a}-h-j-1\right) !} \\
& =\frac{1}{j+1}\left(\begin{array}{c}
N_{a} \\
h
\end{array}\right)\left(N_{a}-h\right)\left(\begin{array}{c}
N_{a}-h-1 \\
j
\end{array}\right)=\frac{1}{j+1} \frac{N_{a} !}{h !\left(N_{a}-h\right) !}\left(N_{a}-h\right)\left(\begin{array}{c}
N_{a}-h-1 \\
j
\end{array}\right) \\
& =\frac{1}{j+1} \frac{N_{a} !}{h !\left(N_{a}-h-1\right) !}\left(\begin{array}{c}
N_{a}-h-1 \\
j
\end{array}\right)=\frac{N_{a}}{j+1}\left(\begin{array}{c}
N_{a}-1 \\
h
\end{array}\right)\left(\begin{array}{c}
N_{a}-1-h \\
j
\end{array}\right)
\end{aligned}
$$


TABLE I

Parameter Settings For the VAlidation of M-MOSK

\begin{tabular}{l|l|l|l}
\hline \hline Sets & $\boldsymbol{M}$ & $m$ & $d$ \\
\hline \hline$S T_{1}$ & 4 & 4 & 0.6 \\
\hline$S T_{2}$ & 4 & 4 & 0.4 \\
\hline$S T_{3}$ & 4 & 2 & 0.6 \\
\hline \hline
\end{tabular}

The PMF of $e_{i}(t)$ is also different from (6)

$$
\begin{aligned}
\tilde{\varphi}_{i}(j, t) & =\operatorname{Pr}\left(e_{i}(t)=j\right) \\
& =\frac{1}{M} \sum_{b=1}^{M} \operatorname{Pr}\left(c_{b, i}\left(N_{b}, t\right)=j\right),
\end{aligned}
$$

where $j=0, \ldots, N=\max \left\{N_{1}, \ldots, N_{M}\right\}$. According to (23) and the independence property among all individual counting processes, the PMF of the total counting process is given by

$$
\begin{aligned}
\varphi_{a, T, m} & \left(N_{a}, k, t\right)=\varphi_{a}\left(N_{a}, k, t\right) \\
& *\left[\tilde{\varphi}_{1}(k, t) * \ldots * \tilde{\varphi}_{m}(k, t)\right],
\end{aligned}
$$

where $a \in 1, \ldots, M$ and $k \in 0, \ldots, N_{a}+m N$. Similar to (1), $\varphi_{a}\left(N_{a}, l, t\right)$ obeys the following expression:

$$
\begin{aligned}
\varphi_{a}\left(N_{a}, l, t\right) & =\operatorname{Pr}\left(c_{a}\left(N_{a}, t\right)=l\right) \\
& =\left(\begin{array}{c}
N_{a} \\
l
\end{array}\right) F_{\psi}^{l}(t)\left[1-F_{\psi}(t)\right]^{N_{a}-l},
\end{aligned}
$$

where $l \in\left[0, N_{a}\right], t \geq 0$. Note that in the M-CSK scheme, detection always takes place at $t=\tau$, when the total counting process is "evaluated". Thus, $\tau$ is the deterministic delay. However, this detection may result in error if the amount of detected molecules falls outside the thresholds corresponding to the symbol being transmitted ( $\sigma_{a 1}$ and $\sigma_{a 2}$ for symbol $a$ ). Hence, the symbol error probability when transmitting symbol $S_{a}$ obeys this equation:

$$
\begin{aligned}
\operatorname{SEP}_{a} & =\operatorname{Pr}\left(c_{a, T, m}\left(N_{a}, \tau\right) \notin\left[\sigma_{a 1}, \sigma_{a 2}\right]\right) \\
& =1-\operatorname{Pr}\left(c_{a, T, m}\left(N_{a}, \tau\right) \in\left[\sigma_{a 1}, \sigma_{a 2}\right]\right) \\
& =1-\sum_{k=\sigma_{a 1}}^{\sigma_{a 2}} \varphi_{a, T, m}\left(N_{a}, k, \tau\right)
\end{aligned}
$$

In general, we have:

$$
\mathrm{SEP}=\frac{1}{M} \sum_{a=1}^{M} \mathrm{SEP}_{a}
$$

\section{VALIDATION OF THE DELAY AND PERFORMANCE ANALYSIS}

In this section, the symbol error probabilities of the M-MOSK and M-CSK schemes in (16) and (29) are validated through extensive simulation experiments. In the simulation experiments, the time step of the diffusing molecules is set to $t_{s}=10^{-6}$ and, the step length $\rho$ of the molecules is set to

$$
\rho=\sqrt{2 t_{s} D} .
$$

The three different parameter settings called as $S T_{i}, i \in\{1,2,3\}$ are used for the validation of the

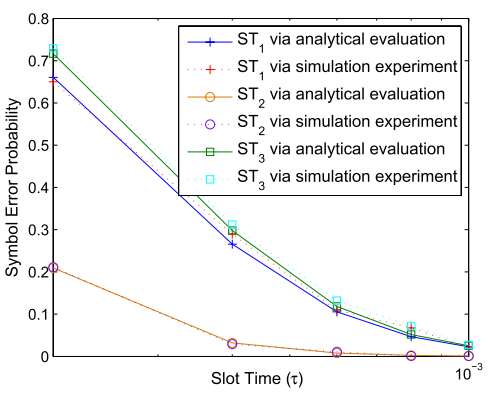

Fig. 3. The validation of the M-MOSK scheme is shown for the three different parameter settings by comparing the analytical SEP expression given in (16) with the SEP values obtained through the simulation experiments.)

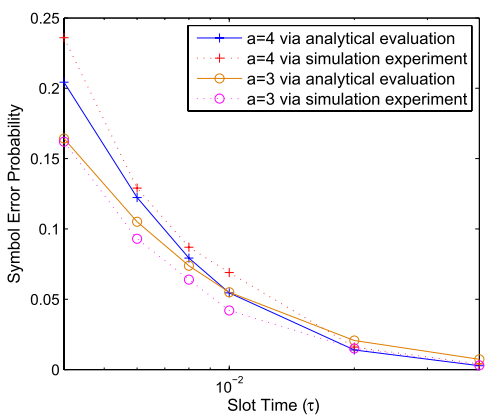

Fig. 4. The validation of the M-CSK scheme is shown for two different scenarios by comparing the analytical SEP expression given in (29) with the SEP values obtained through the simulation experiments. In the first scenario, the current symbol is assumed to be symbol 4 while the current symbol is assumed to be 3 in the second scenario

M-MOSK scheme. In these sets, the parameters $D_{\psi_{a}}=$ $\left\{1000,1100,1200,1300 \mu \mathrm{m}^{2} / \mathrm{s}\right\}, N_{a}=\{20,22,24,26\}$ and $\sigma_{a}=\{10,10,10,10\}$ for $a=1,2,3,4$ are used. However, the parameters $M, m$, and $d$ are changed as given in Table I. In Fig. 3, the symbol error probability in (16) obtained through the derived symbol error probability and the simulation experiments is shown for each parameter settings, i.e., $\left(S T_{i}, i \in\{1,2,3\}\right)$. The SEP values obtained through the analytical expressions and the simulation experiments are very close to each other. This reveals that the analytical derivations are valid. For the validation of the symbol error probability in (29) of the M-CSK scheme, the four different symbols $(M=4)$ are considered with the emission levels $N_{1}=10, N_{2}=20, N_{3}=30, N_{4}=40$ and the corresponding thresholds $\sigma_{1}=15, \sigma_{2}=24$ and $\sigma_{3}=33$. The memory level, the diffusion coefficient, and the distance between the TN and RN are set to $m=4, D=1000 \mu \mathrm{m}^{2} / \mathrm{s}$ and $d=0.6 \mu \mathrm{m}$, respectively. In Fig. 4, two different scenarios are evaluated. In the first scenario, the current symbol is assumed to be symbol 4 and in the second one, the current symbol is assumed to be symbol 3. For these scenarios, the analytical evaluation of SEP in (29) closely follows the SEP values obtained through the simulation experiments. This validates the analytical results derived for the performance of the M-CSK scheme.

\section{Selection of Detection Thresholds}

Here, we introduce two mechanisms to select the thresholds in the M-MOSK and M-CSK schemes. 


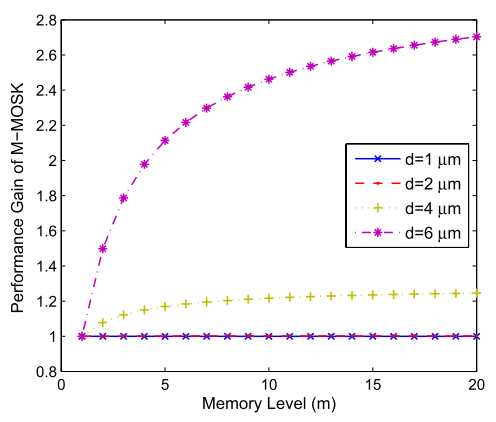

(a)

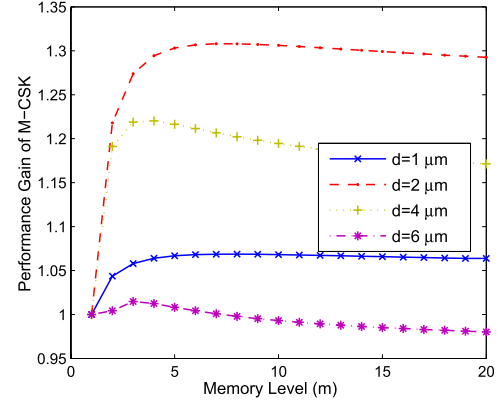

(b)

Fig. 5. By using the performance gain metric given in (36), the effects of the channel memory on the performances of the M-MOSK scheme in (a) and the M-CSK scheme in (b) are shown.

\section{A. Selection of Thresholds in M-MOSK}

In the M-MOSK scheme, a threshold selection method that leads to low symbol error probability values consists of the following:

1) Select emission levels in such a way that the distributions $\varphi_{a}\left(N_{a}, k, \tau\right)$ are similar for all $a=1, \ldots, M$ recall expression (1) in the paper. Since we do not use information about the nature of the reactions that the different types of molecules cause into the receiver nanomachine, nor how the emission levels affect such reactions, a possible criterion is to select emission levels so as to make all counting processes statistically equivalent.

2) Evaluate the average and standard deviation of the counting process associated to each molecule type:

$$
\begin{aligned}
E_{a} & =\sum_{k=0}^{(m+1) N_{a}} k \varphi_{a, T, m}\left(N_{a}, k, \tau\right) \\
S T D_{a} & =\sqrt{\sum_{k=0}^{(m+1) N_{a}}\left(k-E_{a}\right)^{2} \varphi_{a, T, m}\left(N_{a}, k, \tau\right)}
\end{aligned}
$$

3) Select the thresholds using the following alternatives:

- $t h_{a}=\left[E_{a}-S T D_{a}\right], \forall a=1, \ldots, M$

- $t h_{a}=\left[E_{a}-2 S T D_{a}\right], \forall a=1, \ldots, M$

- $t h_{a}=\left[E_{a}-3 S T D_{a}\right], \forall a=1, \ldots, M$

The given three selection alternatives can improve the performance with respect to arbitrary selected threshold values. This improvement is shown and discussed in Section VI.

\section{B. Selection of Thresholds in M-CSK}

For the threshold selection, in the M-CSK scheme, we introduce the reference values $r_{1}, \ldots, r_{M}$ as follows:

$$
r_{a}=E\left(c_{a, T, m}\left(N_{a}, \tau\right)\right), \quad a=1, \ldots, M
$$

where $E($.$) stands for expectation and c_{a, T, m}\left(N_{a}, \tau\right)$ corresponds to (23), evaluated for $t=\tau$. Specifically, the expectation can be calculated as follows:

$$
E\left(c_{a, T, m}\left(N_{a}, \tau\right)\right)=\sum_{k=0}^{N_{a}+m N} k \varphi_{a, T, m}\left(N_{a}, k, \tau\right)
$$

Recall that $N=\max \left\{N_{a}, a=1, \ldots, M\right\}$. Then, the thresholds $t h_{1}, \ldots, t h_{M-1}$ are defined as follows:

$$
t h_{a}=\left[\frac{r_{a}+r_{a+1}}{2}\right], \quad a=1, \ldots, M-1
$$

Then, if $n$ is the number of received molecules, and $\hat{S}$ denotes the selected symbol, the decision rule can be formulated as follows:

$$
\text { If } n \in\left[t h_{a-1}+1, t h_{a}\right] \Rightarrow \hat{S}=S_{a}, \quad \forall a=1, \ldots, M
$$

The given selection mechanism can improve the performance with respect to arbitrary threshold values. This improvement is discussed in Section VI.

\section{Performance Evaluations}

In this section, the performance of both the M-MOSK and M-CSK schemes is evaluated through the analytical expressions of the delay distribution and symbol error probability that have just been validated in the preceding section.

\section{A. Effect of Memory on Performances of M-MOSK and M-CSK Schemes}

In this section, we investigate how the memory level affects the performance of the M-MOSK and M-CSK schemes. To this end, we introduce the following performance gain metric:

$$
\eta(i)=\frac{1-\operatorname{SEP}(i)}{1-\operatorname{SEP}(1)}
$$

$\operatorname{SEP}(i)$ In this formula, $\operatorname{SEP}(i)$ denotes the symbol error probability for the case in which the memory level is $i(m=i)$. Clearly, SEP(1) denotes the symbol error probability for $m=1$. The numerator of $\eta(i)$ represents the probability of successful symbol delivery for $m=i$ while the denominator represents the probability of successful symbol delivery for $m=1$. So, a value $\eta(i)>1$ and progressively increasing with $i$ indicates a performance improvement with memory. By using $\eta(i)$, the effect of memory level on the performance of the M-MOSK and M-CSK schemes are shown in Fig. 5. As it can be noticed, $\eta(i)$ is shown with varying memory levels for different distance values between the transmitter and receiver. An improvement of performance with memory 


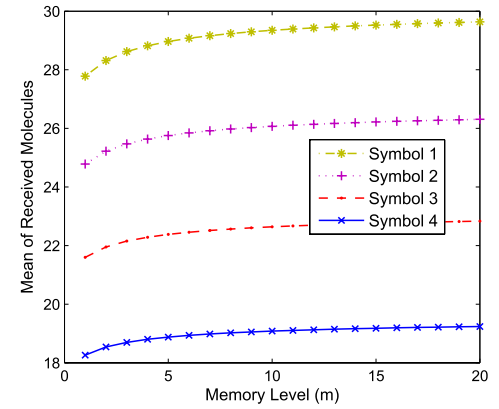

(a)

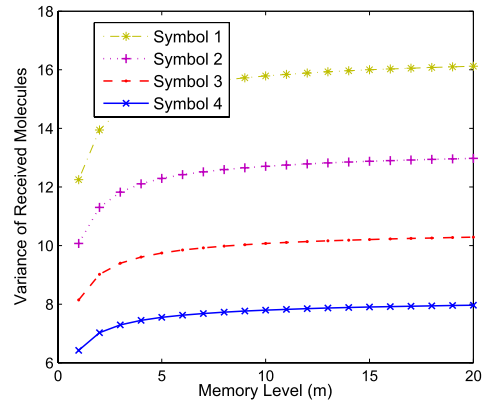

(b)

Fig. 6. The mean (a) and the variance (b) of the received number of molecules for four symbols with the emission levels $40,35,30,25$, are shown for varying levels of channel memory, in the M-MOSK scheme.

is observed, with such improvement much larger for the large distance. The dependence on distance is consistent with the general perception that molecular channels with longer ranges are subject to larger effects of molecular persistence. However, the relevant fact is that such effects are positive. The reason is that, for a relatively wide margin of distances (at least from 2 to $6 \mu \mathrm{m}$ ), the positive effects of previously transmitted same symbols counteract the negative effects of previously transmitted different symbols. However, for the M-MOSK scheme with short distances such as $d=1 \mu \mathrm{m}$, the performance gain hardly changes (in contrast to what happens with larger distances). As in the M-MOSK case, distance plays a major role in the evaluation of memory effects for the M-CSK scheme. However, given the fact that the decision process in the M-CSK scheme is based on two consecutive thresholds per symbol, the evolution of the amount of received molecules from the lower to the upper threshold as long as distance varies, causes a fluctuation on the balance between positive and negative effects. For example, for $d=6 \mu \mathrm{m}$, a very slight negative effect (the decreasing the performance gain from 1 to 0.9802 as the memory level changes from 1 to 20) is observed.

In Fig.6, how the mean and variances of the received number of molecules are changed as the memory level increases is shown by using four symbols (i.e. $M=4$ ) with the emission levels 40, 35, 30 and 25 in the M-MOSK scheme. Please note that in the previous plots, we have used a different set of emission levels (20, 22, 24, 26 in Fig. 3). In order to show the impact of emission level selection, we use the more separated emission levels 40, 35, 30 and 25 for the four symbols. As observed in the figure, the mean and variance values increase with the emission level. That is, the symbol with high emission level has higher mean and variance. While the memory level increases from 1 to 3 , the mean and variance values increase more quickly. As the memory level is further increased, they increase very slightly. In general, as observed, the memory level of the channel has a minor effect on the mean and variance of the received molecules. In order to show how these mean and variance characteristics impact on the symbol error probabilities, in Fig. 7, the symbol error probabilities are shown for the same scenarios used in Fig.6. The threshold values for the four symbols are set

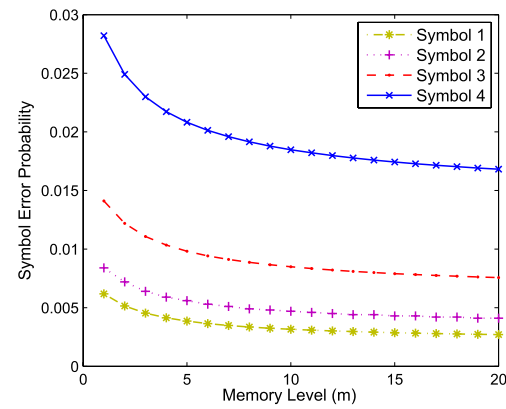

Fig. 7. The symbol error probabilities of four symbols with the emission levels $40,35,30,25$ and the thresholds $20,18,16,14$, respectively, are shown for varying levels of channel memory, in M-MOSK.

as $20,18,16$ and 14 . The symbol error probability increases as the emission level decreases. This is because it is more likely for the molecules associated with the symbols having higher mean and variance to pass the predefined threshold. Therefore, the symbol with the maximum emission level, mean and variance has the minimum error probability.

\section{B. Effect of Threshold on Performance of M-CSK}

Here, we show the effect of threshold selection on the performance of the M-MOSK and M-CSK schemes. For the threshold selection, we use a criterion explained in Section V. In Fig. 8, the effect of the threshold selection mechanism on the performance of the M-MOSK scheme is shown. The selection mechanism generates the thresholds $\{13,14,16,18\}\{14,16,18,19\},\{15,17,18,20\}$, $\{15,17,19,21\},\{15,17,19,21\}$ while we set the arbitrary threshold set as $\{18,18,18,18\}$. As observed in Fig. 8, the thresholds provided by the selection mechanism significantly improve the performance with respect to the arbitrary selection.

In order to show the efficiency of the threshold selection in the M-CSK scheme, in Fig. 8, the average symbol error probability is shown with the selected thresholds and with an arbitrary threshold set. Based on the selection criteria, i.e., $t h_{a}=\left[E_{a}-2 S T D_{a}\right], \forall a$, explained in Section V-B, the threshold selection scheme defined above generates the 


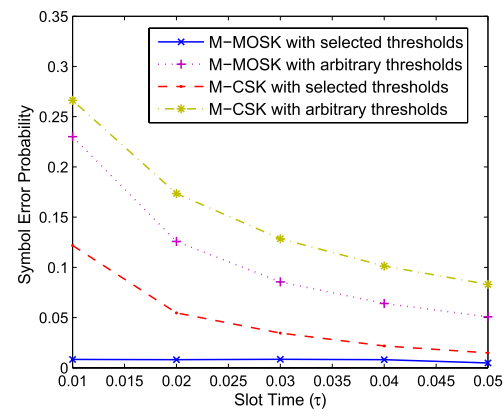

Fig. 8. The effects of threshold on the symbol error probability for both M-MOSK and M-CSK schemes are shown by using the thresholds generated by the threshold selection mechanism and arbitrarily generated thresholds.

five threshold set as the slot time changes. These are $\{15,23,31\},\{15,23,32\},\{15,24,33\},\{15,24,33\}$ and $\{15,24,33\}$. By using these threshold sets and the arbitrarily selected set $\{12,24,36\}$, in Fig. $8(b)$, the symbol error probability is shown. The selected threshold provides more than $\% 100$ improvement in the symbol error probability. Hence, this reveals that the threshold selection can positively affect the performance of the M-CSK scheme.

\section{CONCLUSIONS}

In this paper, by taking into account any level of channel memory, the delay and performance analyses of the M-MOSK and M-CSK schemes are given. After developing the probability distributions of the symbol delay, the performance of the M-MOSK and M-CSK schemes are derived. The analyses are also validated through extensive simulation experiments. Furthermore, in order to speed up the computations of the derived probability distributions, an efficient algorithm is introduced. The numerical evaluations reveal that the performance can be improved by properly selecting system setting. For example, it is shown that, in the M-MOSK scheme, by selecting system parameter properly, the performance can be enhanced as the channel memory is increased. Furthermore, it is shown that the M-MOSK scheme outperforms the M-CSK scheme. In our future works, we plan to extend our investigations into the case of noise to obtain a capacity analysis with arbitrary level of memory. Our future works also include the performance evaluation of synchronization-free M-MOSK scheme. Furthermore, we think that an extension to our work could consider a non-deterministic number of emitted molecules at the beginning of a slot; this number could follow a generic random variable, with the Poisson distribution being a particular case.

\section{REFERENCES}

[1] B. Atakan, Molecular Communications and Nanonetworks: From Nature to Practical Systems. New York, NY, USA: Springer, 2014.

[2] T. Nakano, A. W. Eckford, and T. Haraguchi, Molecular Communication. Cambridge, U.K.: Cambridge Univ. Press, 2013.
[3] I. F. Akyildiz, F. Brunetti, and C. Blázquez, "Nanonetworks: A new communication paradigm," Comput. Netw., Int. J. Comput. Telecommun. Netw., vol. 52, no. 12, pp. 2260-2279, Aug. 2008.

[4] T. Nakano, M. J. Moore, F. Wei, A. V. Vasilakos, and J. Shuai, "Molecular communication and networking: Opportunities and challenges," IEEE Trans. Nanobiosci., vol. 11, no. 2, pp. 135-148, Jun. 2012.

[5] I. Llatser, A. Cabellos-Aparicio, and E. Alarcón, "Networking challenges and principles in diffusion-based molecular communication," IEEE Wireless Commun., vol. 19, no. 5, pp. 36-41, Oct. 2012.

[6] M. S. Kuran, T. Tugcu, and B. O. Edis, "Calcium signaling: Overview and research directions of a molecular communication paradigm," IEEE Wireless Commun., vol. 19, no. 5, pp. 20-27, Oct. 2012.

[7] M. Pierobon and I. F. Akyildiz, "Capacity of a diffusion-based molecular communication system with channel memory and molecular noise," IEEE Trans. Inf. Theory, vol. 59, no. 2, pp. 942-954, Feb. 2013.

[8] M. S. Kuran, H. B. Yilmaz, T. Tugcu, and B. Özerman, "Energy model for communication via diffusion in nanonetworks," Nano Commun. Netw., vol. 1, no. 2, pp. 86-95, 2010.

[9] M. S. Kuran, H. B. Yilmaz, T. Tugcu, and I. F. Akyildiz, "Modulation techniques for communication via diffusion in nanonetworks," in Proc. IEEE Int. Conf. Commun., Jun. 2011, pp. 1-5.

[10] M. H. Kabir and K. S. Kwak, "Effect of memory on BER in molecular communication," Electron. Lett., vol. 50, no. 2, pp. 71-72, Jan. 2014.

[11] C. T. Chou, "Extended master equation models for molecular communication networks," IEEE Trans. Nanobiosci., vol. 12, no. 2, pp. 79-92, Jun. 2013.

[12] C. T. Chou, "Impact of receiver reaction mechanisms on the performance of molecular communication networks," IEEE Trans. Nanotechnol., vol. 14, no. 2, pp. 304-317, Mar. 2015.

[13] H. Arjmandi, A. Gohari, M. N. Kenari, and F. Bateni, "Diffusionbased nanonetworking: A new modulation technique and performance analysis," IEEE Commun. Lett., vol. 17, no. 4, pp. 645-648, Apr. 2013.

[14] R. Mosayebi, H. Arjmandi, A. Gohari, M. Nasiri-Kenari, and U. Mitra, "Receivers for diffusion-based molecular communication: Exploiting memory and sampling rate," IEEE J. Sel. Areas Commun., vol. 32, no. 12, pp. 2368-2380, Dec. 2014.

[15] I. Karatzas and S. E. Shreve, Brownian Motion and Stochastic Calculus. New York, NY, USA: Springer, 1991.

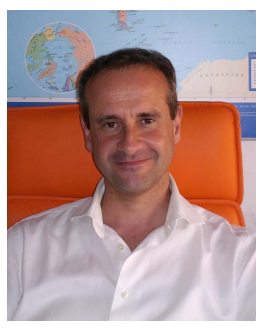

Sebastià Galmés received the M.Sc. degree in electrical engineering from the Polytechnic University of Catalonia, Barcelona, Spain, in 1989, and the $\mathrm{Ph} . \mathrm{D}$. degree in computer science from the University of Balearic Islands, Palma de Mallorca, Spain, in 1999, where he is currently an Associate Professor with the Department of Mathematics and Computer Science. His current research interests focus on wireless communications, wireless sensor networks, and nanoscale and molecular communications.

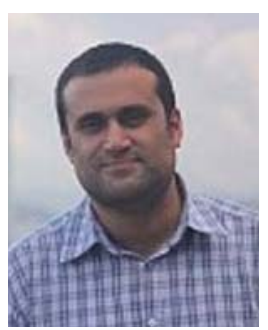

Baris Atakan received the B.Sc. degree from Ankara University, Ankara, Turkey, in 2000, the M.Sc. degree from Middle East Technical University, Ankara, in 2005, and the Ph.D. degree from the Next-Generation and Wireless Communications Laboratory, School of Sciences and Engineering, Koç University, Istanbul, Turkey, in 2011, all in electrical and electronics engineering. He is currently an Associate Professor with the Department of Electrical and Electronics Engineering, İzmir Institute of Technology, İzmir, Turkey. His current research interests include nanoscale and molecular communications, nanonetworks, and biologically inspired communications. 